\title{
Epidemiological and clinical peculiarities of polyglandular syndrome type 3 in pediatric age
}

\author{
Mariella Valenzise*, Tommaso Aversa, Angiola Saccomanno, Filippo De Luca and Giuseppina Salzano
}

\begin{abstract}
Background: Although the autoimmune polyglandular syndrome type 3 (APS-3) is the commonest APS that may be encountered in pediatric age, last year literature includes only few studies aiming to specifically ascertain the clinical spectrum of APS-3 in childhood and adolescence. Aims of these study were: a) to ascertain how many young patients with apparently isolated Hashimoto's thyroiditis $(H T)$ are at risk of manifesting other autoimmune disorders (ADs) compatible with APS-3; b) to individuate the ADs which most frequently segregate with HT in the context of an APS-3.
\end{abstract}

Methods: A selected population of 211 young patients with HT and no risk factors for other APSs was investigated, in order to evaluate the prevalence of other ADs apart from HT and to individuate the ADs which most frequently cluster with HT in the context of APS-3.

Results: The majority of our patients (70.2\%) was found to be affected by no other ADs apart from HT, whereas the remaining $29.8 \%$ were classified as affected by an APS-3. The ADs which were found most frequently in aggregation with HT were type 1 diabetes mellitus T1DM (61.9\%) and celiac disease CD (22.2\%).

Conclusions: 1) About 30\% of young patients with HT may exhibit a clinical picture consistent with APS-3; 2) In the context of APS-3 the ADs that most frequently cluster with HT are T1DM and CD.

Keywords: Hashimoto's thyroiditis, Celiac disease, Type 1 diabetes mellitus

\section{Background}

The autoimmune polyglandular sindromes (APSs) are a group of disorders characterized by the combination of multiple autoimmune diseases (ADs). In two of these conditions, i.e. APS-1 and IPEX syndrome, ADs may also be associated with immunodeficiency [1].

According to the original and historical classification of Neufeld et al. [2], APS-3 is a separate clinical entity, that differs from IPEX syndrome for the absence of immunodeficiency and from the remaining APSs for both the mandatory presence of autoimmune thyroid diseases (AITDs) and the mandatory absence of Addison's disease and hypoparathyroidism [3]. APS-3 is more common than the other APSs and may present even in pediatric age, especially during adolescence and early adulthood

* Correspondence: mvalenzise@unime.it

UOC Pediatria, Department of Human Pathology in Adulthood and

Childhood, University of Messina, via Consolare Valeria, 98125 Messina, Italy
[3]. Other ADs which may be observed in the patients with APS-3 are type 1 diabetes mellitus T1DM (APS$3 \mathrm{~A})$, celiac disease $\mathrm{CD}$, atrophic gastritis or pernicious anemia (APS-3b), vitiligo or alopecia (APS-3c) and rheumatologic diseases (APS-3d).

Although APS-3 is the most common polyglandular syndrome that may be encountered in pediatric age [4], nevertheless there are only few studies, in the pediatric literature, aiming to specifically ascertain the clinical spectrum of this syndrome in childhood and adolescence [4].

In the present cross-sectional study we have investigated, for the first time, the clustering of extra-thyroidal ADs in a selected population of 211 children and adolescents with pre-existing Hashimoto's thyroiditis (HT) and no detectable signs of either Addison's disease or hypoparathyroidism or immunodeficiency, which would not be compatible, by definition, with diagnosis of APS-3 [3]. 
Our aims were: a) to ascertain how many young patients with $\mathrm{HT}$ are at risk of manifesting other ADs compatible with APS-3; b) to individuate the ADs which most frequently segregate with HT in the context of an APS-3.

\section{Methods}

\section{Patients}

The entire study population consisted of 211 young patients (162 girls; median age 11.2 years, range 2.6-22.1), who were admitted to this study according to the following inclusion criteria: a) age $<25$ yrs.; b) well-established diagnosis of HT; c) no clinical and/or biological signs of either immunodeficiency or Addison's disease or hypoparathyroidism at the time of this study.

\section{Design}

All the recruited patients were cross-sectionally investigated, in order to evaluate the prevalence of concomitant extra-thyroidal ADs and to individuate the ADs which most frequently cluster with HT in the context of an APS-3.

\section{Methods}

Diagnosis of HT was assessed according to the following criteria: 1) hypoechogenic thyroid pattern at ultrasonography (US) consistent with AITDs; 2) positive thyroglobulin and/or thyroid peroxidase autoantibodies (TGAbs and TPOAbs, respectively).

TGAb and TPOAb serum concentrations were measured by chemiluminescent immunometric assays. According to these methods, values above 20 or $30 \mathrm{IU} / \mathrm{L}$, respectively, are defined as positive [5-9].

Thyroid US examinations for the assessment of echogenicity were always performed by experienced ultrasonographers with high-resolution US machines.

In our screening approach we took into consideration the following extra-thyroidal ADs: T1DM, CD, pernicious anemia, atrophic gastritis, vitiligo, alopecia areata and idiopathic arthritis. All these diseases were screened by both specific anamnesis and clinical evaluation. Furthermore, all the recruited patients underwent the following autoantibody (Ab) assays: insulin, glutamic acid decarboxylase and islet cell Abs, anti-transglutaminase, anti-gliadin and anti-endomysium Ig G/A, anti-gastric parietal cell Abs and anti-nuclear and anti-smooth muscle Abs. Diagnosis of CD in the patients with specific Ab positivity was confirmed by small intestine biopsy. Diagnosis of both vitiligo and alopecia areata was only based on clinical evaluation $[10,11]$.

$\mathrm{Ab}$ assays were centralized in the same Laboratory of our Department.

The individuals who, at the time of this study, exhibited at least one additional AD, apart from HT, were classified as patients with APS-3 (Group A), whereas those with isolated HT were included in Group B.
The study design was approved by the Ethical Committee of our University Hospital.

\section{Statistical analysis}

It was performed by using SPSS 16.0 version (SPSS, Inc., Chicago, IL, USA).

Frequency rates of the patients with either associated ADs or isolated HT were compared by chi-square test.

Median ages of the patients belonging to Groups A and B were compared between them by Mann-Whitney test.

\section{Results}

In the entire study population the majority of our patients with HT (70.2\%) was not found to be affected by any associated ADs, whilst $28.9 \%$ were found to be affected by only one extrathyroidal AD and $0.9 \%$ were detected to be affected by at least two extra-thyroidal ADs.

On the basis of the established diagnostic criteria 29.8\% of the subjects were labelled as patients with APS-3 (Group A), whereas the remaining 70.2\% were classified as patients with isolated HT (Group B).

Median ages of the patients belonging to Groups A and $B$ were very similar: respectively 12.2 , range $2.6-22.1$ yrs. vs 11.0, range 4.1-18.0. In both groups female gender was equally preponderant: respectively $66.7 \%$ vs $66.9 \%$.

Among the different extra-thyroidal ADs, the one that was detected most commonly in our study population was T1DM (61.9\%), followed by CD (22.2\%), vitiligo (6.3\%), alopecia areata, idiopathic arthritis and atrophic gastritis (1.6\% each). Among these ADs, T1DM was the one that presented earliest, followed by vitiligo and atrophic gastritis (Table 1).

\section{Discussion}

Among the APSs, the one that has been most extensively investigated in the last years is APS-1, i.e. a rare autosomal recessive disorder due to mutations in the AutoImmune Regulator (AIRE) gene [12, 13]. This syndrome is mainly characterized by the classical triad of chronic mucocutaneous syndrome, hypoparathyroidism and Addison's disease [14, 15]. More recently, however, many other autoimmune manifestations have been reported to be possibly observed in this condition [16-20].

By contrast, the remaining APSs have been only sporadically investigated in the last few years, although their prevalences are probably higher than that of APS-1 [4].

To the best of our knowledge, this is the first study aiming to specifically ascertain the prevalence of APS-3 in a pediatric study population with isolated $\mathrm{HT}$ and no risk factors for the remaining APSs. Furthermore, our study design gave us the opportunity of addressing another intriguing issue, i.e. which may be the preferential clustering of extra-thyroidal ADs in the context of APS-3. 
Table 1 Median ages (and ranges) at the presentation of the commonest extra-thyroidal autoimmune disorders that were found in the group of 63 patients with autoimmune polyglandular syndrome type 3

\begin{tabular}{lllllll}
\hline & Type 1 Diabetes Mellitus & Vitiligo & Atrophic Gastritis & Alopecia Areata & Celiac disease & Idiopathic Arthritis \\
\hline Median age & 9.7 & 11.2 & 11.6 & 12.8 & 13.8 & 16.2 \\
Range & $1.6-17.1$ & $6.3-17.3$ & - & - & $1.4-19.0$ & - \\
\hline
\end{tabular}

According to our results, the probability that young patients with isolated HT and no risk factors for the remaining APSs might exhibit other ADs compatible with APS-3 may be estimated around $29.8 \%$, at least at a median age of 11.2 years. However, it cannot be excluded that such risk might furtherly increase with increasing patient age.

In fact, it should be considered that the prevalence of T1DM in the general pediatric population increases with age, achieving its zenith between 15 and 19 years [21]. Considering that the median age of the individuals included in our study population was 11.2 years, it would be reasonable to postulate that younger children might not have developed T1DM yet, but they might become diabetic in older age. Furthermore, it has to be underlined that the remaining extra-thyroidal disorders which were detected in this series presented even later than T1DM.

On the other hand, the pivotal role played by age in affecting the development of extra-thyroidal ADs has been just recently confirmed [9].

\section{Conclusions}

About 30\% of young patients with HT may exhibit a clinical picture consistent with APS-3. In the context of APS-3 the ADs that most frequently cluster with HT are T1DM and CD.

\section{Abbreviations}

ADs: Autoimmune disorders; APS-1: Autoimmune polyglandular syndrome type 1; APS-3: Autoimmune polyglandular syndrome type 3; CD: Celiac disease; HT: Hashimoto's thyroiditis; IPEX: Immunodysregulation polyendocrinopathy enteropathy X-linked syndrome; TGAbs: Thyroglobulin autoantibodies; TPOAbs: Thyroid peroxidase autoantibodies;

US: Ultrasonography

\section{Acknowledgments}

Not applicable.

\section{Funding}

This research did not receive any specific grant.

\section{Availability of data and materials}

Data sharing not applicable to this article as no datasets were generated or analyzed during the current study.

\section{Authors' contributions}

MV and FDL have written the paper; GS has organized the material and prepared its distribution in the different sections; AS has collected the data of the patients, TA has prepared the table and collected references. All authors read and approved the final manuscript. Each Author listed on the manuscript has seen and approved the submission of the present version of the manuscript and takes full responsibility for the manuscript.
Ethics approval and consent to participate

The informed consent has been obtained from the parents of our patients and the study was approved by Ethical Committee of our University Hospital (A.O.U. Policlinico "G. Martino").

\section{Competing interests}

The authors declare that they have no competing interests.

\section{Publisher's Note}

Springer Nature remains neutral with regard to jurisdictional claims in published maps and institutional affiliations.

Received: 25 May 2017 Accepted: 28 July 2017

Published online: 07 August 2017

References

1. Michels AW. Gottlieb PA Autoimmune polyglandular syndromes. Nat Rev Endocrinol. 2010;6:270-7.

2. Neufeld Maclaren N, Blizzard R. Autoimmune polyglandular syndromes. Pediatr Ann. 1980;9:154-62.

3. Betterle C, Zanchetta R. Update on autoimmune polyendocrine syndromes (APS). Acta Biomed. 2003;74:9-33.

4. Kahaly GJ. Polyglandular autoimmune syndromes. Eur J Endocrinol. 2009; 161:11-20.

5. Wasniewska M, Corrias A, Salerno M, Mussa A, Capalbo D, Messina MF, et al. Thyroid function patterns at Hashimoto's thyroiditis presentation in childhood and adolescence are mainly conditioned by patients' age. Horm Res Paediatr. 2012;78:232-6.

6. Wasniewska M, Aversa T, Salerno M, Corrias A, Messina MF, Mussa A, et al. Five-year prospective evaluation of thyroid function in girls with subclinical mild hypothyroidism of different etiology. Eur J Endocrinol. 2015;173:801-8.

7. Aversa T, Corrias A, Salerno M, Tessaris D, Di Mase R, Valenzise M, et al. Fiveyear prospective evaluation of thyroid function test evolution in children with Hashimoto's Thyroiditis presenting with either Euthyroidism or subclinical hypothyroidism. Thyroid. 2016;26:1450-6.

8. Wasniewska M, Salerno M, Corrias A, Mazzanti L, Matarazzo P, Corica D, et al. The evolution of thyroid function after presenting with Hashimoto Thyroiditis is different between initially Euthyroid girls with and those without turner syndrome. Horm Res Paediatr. 2016;86:403-9.

9. Aversa T, Valenzise M, Corrias A, Salerno M, lughetti L, Tessaris D, et al. In children with autoimmune thyroid diseases the association with down syndrome can modify the clustering of extra-thyroidal autoimmune disorders. J Pediatr Endocrinol Metab. 2016;29:1041-6.

10. Ezzedine K, Eleftheriadou V, Whitton M, van Geel N. Vitiligo. Lancet. 2015; 386:74-84.

11. Islam N, Leung PS, Huntley AC, Gershwin ME. The autoimmune basis of alopecia areata: a comprehensive review. Autoimmun Rev. 2015;14:81-9.

12. Cervato S, Mariniello B, Lazzarotto F, Morlin L, Zanchetta R, Radetti G, et al. Evaluation of the autoimmune regulator (AIRE) gene mutations in a cohort of Italian patients with autoimmune-polyendocrinopathy-candidiasisectodermal-dystrophy (APECED) and in their relatives. Clin Endocrinol. 2009; 70:421-8.

13. Valenzise M, Wasniewska M, Mirabelli S, De Luca F, Cervato S, Betterle C. Identification of two novel mutations in the first Sicilian APECED patient with no R203X mutation in AIRE gene and review of Italian APECED genotypes. Gene. 2012;499:343-6.

14. Perheentupa J. Autoimmune polyendocrinopathy-candidiasis-ectodermal dystrophy. J Clin Endocrinol Metab. 2006;91:2843-50.

15. Valenzise M, Fierabracci A, Cappa M, Porcelli P, Barcellona R, De Luca F, et al. Autoimmune polyendocrinopathy-candidiasis-ectodermal dystrophy: report 
of seven additional sicilian patients and overview of the overall series from Sicily. Horm Res Paediatr. 2014;82:127-32.

16. De Luca F, Valenzise M, Alaggio R, Arrigo T, Crisafulli G, Salzano G, et al. Sicilian family with autoimmune polyendocrinopathy-candidiasis-ectodermal dystrophy (APECED) and lethal lung disease in one of the affected brothers. Eur J Pediatr. 2008;167:1283-8.

17. Valenzise M, Meloni A, Betterle C, Giometto B, Autunno M, Mazzeo A, et al. Chronic inflammatory demyelinating polyneuropathy as a possible novel component of autoimmune poly-endocrine-candidiasis-ectodermal dystrophy. Eur J Pediatr. 2009;168:237-40.

18. Su MA, Davini D, Cheng P, Giang K, Fan U, DeVoss JJ, et al. Defective autoimmune regulator-dependent central tolerance to myelin protein zero is linked to autoimmune peripheral neuropathy. J Immunol. 2012;188:4906-12.

19. Valenzise M, Alessi L, Bruno E, Cama V, Costanzo D, Genovese C, et al. APECED syndrome in childhood: clinical spectrum is enlarging. Minerva Pediatr. 2016;68:226-9.

20. Valenzise M, Aversa T, Salzano G, Zirilli G, De Luca F, Su M. Novel insight into chronic inflammatory Demyelinating Polineuropathy in APECED syndrome: molecular mechanisms and clinical implications in children. Ital J Pediatr. 2017:43:11.

21. Pettitt DJ, Talton J, Dabelea D, Divers J, Imperatore G, Lawrence JM, et al. Prevalence of diabetes in US youth in 2009: the SEARCH for diabetes in youth study. Diabetes Care. 2014;37:402-8.

\section{Submit your next manuscript to BioMed Central} and we will help you at every step:

- We accept pre-submission inquiries

- Our selector tool helps you to find the most relevant journal

- We provide round the clock customer support

- Convenient online submission

- Thorough peer review

- Inclusion in PubMed and all major indexing services

- Maximum visibility for your research

Submit your manuscript at www.biomedcentral.com/submit 